\title{
The Preconditioner of GPHSS Method for Saddle Point Systems
}

\author{
Shiheng Wang ${ }^{1}$ and Ke Wang ${ }^{2, a}$ * \\ ${ }^{1}$ Department of Automotive Engineering, Nanyang Vocational College of Agriculture, \\ Nanyang 473000, P.R. China \\ ${ }^{2}$ Department of Mathematics, College of Sciences, Shanghai 200444, P.R. China \\ akwang@shu.edu.cn \\ ${ }^{*}$ corresponding author
}

Keywords: Saddle point problem, GHPSS method, Preconditioner

Abstract. Pan and Wang presented a generalized preconditioned Hermitian and skew-Hermitian splitting (GPHSS) method [J. Numer. Methods Comput. Appl., 32., 174-182, 2011] for saddle point problems. The method is improved to solve saddle point systems whose $(1,1)$ block is a symmetric positive definite $M$-matrix with a new choice of the preconditioner and compared with other preconditioners. The results show that the new preconditioner outperforms the previous ones.

\section{Introduction}

For the saddle point problem (augmented system)

$$
\left(\begin{array}{cc}
A & B \\
B^{\mathrm{T}} & 0
\end{array}\right)\left(\begin{array}{l}
x \\
y
\end{array}\right)=\left(\begin{array}{l}
p \\
q
\end{array}\right)
$$

where $A \in R^{m \times m}$ is a symmetric positive definite matrix, $B \in R^{m \times n}(m>n)$ is of full column rank, $p \in R^{m}$ and $q \in R^{n}$, there are many kinds of iterative methods such as Uzawa [3], SOR-like [4], GSI [6], GAOR [8], HSS [1] and PHSS [2]. Based on the PHSS method, Pan and Wang [7] suggested a GPHSS method with two parameters. In this paper, we further study the GPHSS method to improve it for solving such saddle point systems whose $(1,1)$ block is a symmetric positive definite $M$-matrix with a new choice of the preconditioner and compare it with other preconditioners.

The rest of the paper is organized as follows. In Section 2, the GPHSS method is briefly introduced. In Section 3, we discuss the new choice of the preconditioner. In Section 4, we give numerical examples to show the improvement is efficient. The conclusions are presented in Section 5.

\section{GPHSS Method for Saddle Point Problem}

In this section, we briefly introduce the GPHSS method for saddle point problems.

System (1) can be rewritten as [4]

where

$$
\text { A } z=b
$$

$$
A=\left(\begin{array}{cc}
A & B \\
-B^{\mathrm{T}} & 0
\end{array}\right), z=\left(\begin{array}{c}
x \\
y
\end{array}\right), b=\left(\begin{array}{c}
p \\
-q
\end{array}\right) .
$$

Define matrix $P=\left(\begin{array}{cc}A & 0 \\ 0 & Q\end{array}\right)$, where $Q \in R^{n \times n}$ is nonsingular and symmetric, referred to the preconditioning parameter matrix.

Given the initial vectors $x^{(0)} \in R^{m}, y^{(0)} \in R^{m}$, as well as the relaxation factors $\omega>0$ and $\tau>0$, for $k=0,1,2, \mathrm{~L}$, till the iteration sequence $\left(x^{(k)^{\mathrm{T}}}, y^{(k)^{\mathrm{T}}}\right)^{\mathrm{T}}$ converges, Pan and Wang [7] proposed the GPHSS algorithm as followed: 


$$
\begin{aligned}
& x^{\left(k+\frac{1}{2}\right)}=\frac{\omega}{1+\omega} x^{(k)}+\frac{1}{1+\omega} A^{-1}\left(p-B y^{(k)}\right), \\
& y^{\left(k+\frac{1}{2}\right)}=y^{(k)}+\frac{1}{\tau} Q^{-1}\left(B^{\mathrm{T}} x^{(k)}-q\right), \\
& y^{(k+1)}=\tau D^{-1} Q y^{\left(k+\frac{1}{2}\right)}+D^{-1}\left(\left(1-\frac{1}{\omega}\right) B^{\mathrm{T}} x^{\left(k+\frac{1}{2}\right)}+\frac{1}{\omega} B^{\mathrm{T}} A^{-1} p-q\right), \\
& x^{(k+1)}=\frac{\omega-1}{\omega} x^{\left(k+\frac{1}{2}\right)}+\frac{1}{\omega} A^{-1}\left(p-B y^{(k+1)}\right),
\end{aligned}
$$

where $D=\omega^{-1} B^{\mathrm{T}} A^{-1} B+\tau Q \in R^{n \times n}$. When $\omega=\tau$, GPHSS algorithm is the PHSS algorithm.

Denote $H=\frac{1}{2}\left(A+A^{\mathrm{T}}\right), S=\frac{1}{2}\left(A^{A}-A^{\mathrm{T}}\right)$, then the matrix form of the GPHSS is as follows:

where

$$
\left\{\begin{array}{l}
(\Omega P+H)\left(\begin{array}{l}
x^{\left(k+\frac{1}{2}\right)} \\
y^{\left(k+\frac{1}{2}\right)}
\end{array}\right)=(\Omega P-S)\left(\begin{array}{l}
x^{(k)} \\
y^{(k)}
\end{array}\right)+b, \\
(\Omega P+S)\left(\begin{array}{l}
x^{(k+1)} \\
y^{(k+1)}
\end{array}\right)=(\Omega P-H)\left(\begin{array}{l}
x^{\left(k+\frac{1}{2}\right)} \\
y^{\left(k+\frac{1}{2}\right)}
\end{array}\right)+b,
\end{array}\right.
$$

$$
\Omega=\left(\begin{array}{cc}
\omega I_{m} & 0 \\
0 & \tau I_{n}
\end{array}\right)
$$

and $I_{m}$ and $I_{n}$ are $m \times m$ and $n \times n$ identity matrices. The iterative matrix is

$$
M_{(\omega, \tau)}=(\Omega P+S)^{-1}(\Omega P-H)(\Omega P+H)^{-1}(\Omega P-S) .
$$

\section{New Choice of the Preconditioner}

Consider $m \times m$ symmetric matrices $T=\left(t_{i j}\right)$ with negative off-diagonal elements and positive row (and column) sums, i.e.,

$$
\begin{cases}t_{i i}>0, & i=1, \mathrm{~L}, m, \\ t_{i j}=t_{j i}<0, & i \neq j, \\ \sum_{k=1}^{m} t_{i k}>0, & i=1, \mathrm{~L}, m .\end{cases}
$$

Such matrices are positive definite and thus are $M$-matrices.

Let $S=\left(s_{i j}\right)$ be the $m \times m$ matrix defined in [5] as $s_{i j}=\frac{\delta_{i j}}{t_{i j}}+\frac{1}{t}$, where $\delta_{i j}$ is the Kronecker delta function $\delta_{i j}=\left\{\begin{array}{ll}1, & i=j, \\ 0, & i \neq j,\end{array}\right.$ and $\bar{t}=\sum_{i=1}^{m} \sum_{j=1}^{m} t_{i j}$ with $t_{i j}$ as in (4). From [5], we see that the matrix $S$ is symmetric positive definite and $S$ has the form

$$
S=\frac{1}{t}\left(\begin{array}{l}
1 \\
1 \\
\mathrm{M} \\
1
\end{array}\right) \cdot(1,1, \mathrm{~L}, 1)+\operatorname{diag}\left(1 / t_{11}, 1 / t_{22}, \mathrm{~L}, 1 / t_{m m}\right) .
$$

For augmented system (1) with matrix $A$ satisfying (4), we can take $T=A$ and the preconditioning parameter matrix $Q=B^{\mathrm{T}} S B$ in the GPHSS method. We have the following results.

Theorem If $A=\left(a_{i j}\right)_{m \times m}$ in (1) satisfies (4), taking $T=A, S$ as (5) and $Q=B^{\mathrm{T}} S B$, then $Q$ is symmetric and positive definite, thus the GPHSS method (3) is convergent with $\omega>0$ and $\tau>0$.

Proof The results can directly obtained from Theorems 1 and 2 in [7]. 


\section{Numerical Experiments}

In this section, we give three examples to illustrate the GPHSS method with the new choice of the preconditioner. All performances are taken in MATLAB 7.12 with $2.70 \mathrm{GHz}$ CPU, 4.00GB RAM and Windows 7 Professional. In our experiments, the initial guess is 0 and the stopping criterion is

$$
\frac{\left\|r^{(k)}\right\|_{2}}{\left\|r^{(0)}\right\|_{2}}<10^{-6},
$$

where $r^{(k)}$ is the residual vector after k iterations, $Q=B^{\mathrm{T}} B$ and the parameters $\omega=\omega^{*}, \tau=\tau^{*}$. The results are listed in Tables 1-3.

Example 1 Consider $(m+n) \times(m+n)$ augmented system (1) with

$$
A=\left(a_{i j}\right)_{m \times m}=\left\{\begin{array}{ll}
a_{i j}=i+j, & i=j, \\
a_{i j}=-\frac{1}{m}, & i \neq j,
\end{array} \quad 1 \leq i, j \leq m,\right.
$$

$B=\operatorname{eye}(m, n), b=(1,1, \ldots, 1)^{\mathrm{T}}$ and $q=(0,0, \ldots, 0)^{\mathrm{T}}$.

Table 1

Iterations (IT), CPU time (t) and relative error (ERR) for Example 1

\begin{tabular}{r|r|rrr|rrr|rcc}
\hline \multirow{2}{*}{$m$} & \multirow{3}{*}{$n$} & \multicolumn{3}{|c|}{$Q=B^{\mathrm{T}} B$} & \multicolumn{3}{c|}{$Q=\frac{1}{2} I$} & \multicolumn{3}{c}{$Q=B^{\mathrm{T}} S B$} \\
\cline { 3 - 11 } & & IT & $t$ & ERR & IT & $t$ & ERR & IT & $t$ & ERR \\
\hline 50 & 40 & 21 & 0.04 & $6.3 \mathrm{e}-7$ & 21 & 0.03 & $6.3 \mathrm{e}-7$ & 8 & 0.00 & $1.5 \mathrm{e}-7$ \\
200 & 150 & 30 & 0.44 & $7.8 \mathrm{e}-7$ & 30 & 0.42 & $7.8 \mathrm{e}-7$ & 8 & 0.14 & $1.4 \mathrm{e}-7$ \\
400 & 300 & 36 & 3.20 & $9.5 \mathrm{e}-7$ & 36 & 3.17 & $9.5 \mathrm{e}-7$ & 8 & 0.72 & $1.9 \mathrm{e}-7$ \\
500 & 400 & 39 & 6.19 & $9.5 \mathrm{e}-7$ & 39 & 5.97 & $9.5 \mathrm{e}-7$ & 8 & 1.29 & $4.4 \mathrm{e}-7$ \\
700 & 500 & 42 & 14.91 & $7.8 \mathrm{e}-7$ & 42 & 14.13 & $7.8 \mathrm{e}-7$ & 8 & 2.91 & $1.4 \mathrm{e}-7$ \\
1000 & 700 & 46 & 42.45 & $8.2 \mathrm{e}-7$ & 46 & 41.67 & $8.2 \mathrm{e}-7$ & 8 & 7.50 & $1.3 \mathrm{e}-7$ \\
\hline
\end{tabular}

Example 2 Consider $(m+n) \times(m+n)$ augmented system (1) with

$$
A=\left(a_{i j}\right)_{m \times m}= \begin{cases}a_{i j}=-\frac{1}{2}-\frac{i}{2 m}, & i<j, \\ a_{i j}=a_{j i}, & i>j, \quad 1 \leq i, j \leq m, \\ a_{i j}=-\sum a_{i k}+1+\frac{i}{m}, & i=j,\end{cases}
$$

$B=(1 / 2) * \operatorname{eye}(m, n), b=(1,0, \ldots, 0)^{\mathrm{T}}$ and $q=(1,0, \ldots, 0)^{\mathrm{T}}$.

Table 2

Iterations (IT), CPU time (t) and relative error (ERR) for Example 1

\begin{tabular}{r|r|rrc|rcc|ccc}
\hline \multirow{2}{*}{$m$} & \multirow{3}{*}{$n$} & \multicolumn{4}{|c|}{$Q=B^{\mathrm{T}} B$} & \multicolumn{3}{c|}{$Q=\frac{1}{2} I$} & \multicolumn{3}{c}{$Q=B^{\mathrm{T}} S B$} \\
\cline { 3 - 11 } & & IT & $t$ & ERR & IT & $t$ & ERR & IT & $t$ & ERR \\
\hline 50 & 40 & 18 & 0.03 & $7.1 \mathrm{e}-7$ & 18 & 0.03 & $6.3 \mathrm{e}-7$ & 5 & 0.00 & $1.6 \mathrm{e}-8$ \\
200 & 150 & 29 & 0.44 & $4.7 \mathrm{e}-7$ & 29 & 0.45 & $7.8 \mathrm{e}-7$ & 4 & 0.08 & $7.6 \mathrm{e}-7$ \\
400 & 300 & 35 & 2.79 & $7.3 \mathrm{e}-7$ & 35 & 2.99 & $9.5 \mathrm{e}-7$ & 4 & 0.44 & $6.9 \mathrm{e}-7$ \\
500 & 400 & 38 & 5.85 & $5.8 \mathrm{e}-7$ & 38 & 6.05 & $9.5 \mathrm{e}-7$ & 5 & 0.87 & $6.6 \mathrm{e}-9$ \\
700 & 500 & 41 & 13.51 & $5.1 \mathrm{e}-7$ & 41 & 14.06 & $7.8 \mathrm{e}-7$ & 5 & 1.89 & $5.4 \mathrm{e}-9$ \\
1000 & 700 & 45 & 41.25 & $5.5 \mathrm{e}-7$ & 45 & 42.49 & $8.2 \mathrm{e}-7$ & 5 & 4.82 & $1.1 \mathrm{e}-8$ \\
\hline
\end{tabular}

Example 3 Consider $(m+n) \times(m+n)$ augmented system (1) with 


$$
\begin{aligned}
& A=\left(a_{i j}\right)_{m \times m}= \begin{cases}a_{i j}=i+1, & i=j, \\
a_{i j}=1, & |i-j|=1, \quad 1 \leq i, j \leq m, \\
a_{i j}=0, & \text { others, }\end{cases} \\
& B=\left(b_{i j}\right)_{m \times m}=\left\{\begin{array}{ll}
b_{i j}=j, & i=j+m-n, \\
b_{i j}=0, & \text { others, }
\end{array} \quad 1 \leq i \leq m, 1 \leq j \leq n,\right.
\end{aligned}
$$

$b=(1,0, \ldots, 0)^{\mathrm{T}}$ and $q=(1,0, \ldots, 0)^{\mathrm{T}}$.

Table 3

\begin{tabular}{|c|c|c|c|c|c|c|c|c|c|c|}
\hline \multirow{2}{*}{$m$} & \multirow{2}{*}{$n$} & \multicolumn{3}{|c|}{$Q=B^{\mathrm{T}} B$} & \multicolumn{3}{|c|}{$Q=\frac{1}{2} I$} & \multicolumn{3}{|c|}{$Q=B^{\mathrm{T}} S B$} \\
\hline & & IT & $t$ & ERR & IT & $t$ & ERR & IT & $t$ & ERR \\
\hline 50 & 40 & 12 & 0.03 & $3.0 \mathrm{e}-7$ & 41 & 0.06 & $7.6 \mathrm{e}-7$ & 8 & 0.00 & $1.6 \mathrm{e}-8$ \\
\hline 200 & 150 & 12 & 0.22 & $3.2 \mathrm{e}-7$ & 88 & 1.72 & $9.5 e-7$ & 8 & 0.12 & $7.6 \mathrm{e}-7$ \\
\hline 400 & 300 & 12 & 1.44 & $7.6 \mathrm{e}-7$ & 129 & 14.99 & $8.8 \mathrm{e}-7$ & 8 & 0.66 & $6.9 \mathrm{e}-7$ \\
\hline 500 & 400 & 13 & 2.94 & $1.0 \mathrm{e}-7$ & 141 & 29.09 & $8.7 \mathrm{e}-7$ & 8 & 1.31 & $6.6 e-9$ \\
\hline 700 & 500 & 12 & 5.12 & $5.2 \mathrm{e}-7$ & 177 & 72.29 & $9.6 e-7$ & 9 & 3.39 & $5.4 \mathrm{e}-9$ \\
\hline 1000 & 700 & 12 & 12.39 & $5.2 \mathrm{e}-7$ & 216 & 221.60 & $9.6 \mathrm{e}-7$ & 9 & 9.55 & $1.1 \mathrm{e}-8$ \\
\hline
\end{tabular}

Iterations (IT), CPU time (t) and relative error (ERR) for Example 1

Tables 1, 2 and 3 give the number of iterations required for convergence, CPU time and relative error, which show that the new choice of the preconditioner $B^{\mathrm{T}} S B$ is much better than $B^{\mathrm{T}} B$ and $\alpha I$ with the optimal parameters $\omega^{*}$ and $\tau^{*}$.

\section{Conclusion}

In this paper, a new choice of the preconditioner $B^{\mathrm{T}} S B$ is presentedto accelerate the GPHSS method suggested by Pan and Wang [7] for solving saddle point problems with $A$ is a positive definite $M$-matrix satisfying (4). With the new choice, we can avoid computing $A^{-1}$ and obtain a good approximation of the choice $B^{\mathrm{T}} A^{-1} B$. Thus, the method with the new choice is very fast. Numerical experiments show that the new choice of the parameter matrix are better than $B^{\mathrm{T}} B$ and $\alpha I$ which are common choices for solving saddle point problems and the number of iterations is almost constant.

\section{Acknowledgments}

The second author was supported by National Natural Science Foundation of China (11301330) and the grant "The First-class Discipline of Universities in Shanghai".

\section{References}

[1] Z.-Z. Bai, G.H. Golub, M.K. Ng, Hermitian and skew-Hermitian splitting methods for non-Hermitian positive definite linear systems, SIAM J. Matrix Anal. Appl. 24 (2003) 603-626.

[2] Z.-Z. Bai, G.H. Golub, J.-Y. Pan, Preconditioned Hermitian and skew-Hermitian splitting methods for non-Hermitian positive semidefinite linear systems, Numer. Math. 98 (2004) 1-32.

[3] Y. Cao, Y. Lin, Y. Wei, Nonlinear Uzawa methods for solving nonsymmetric saddle point problems, J. Appl. Math. Comput. 21 (2006) 1-21.

[4] G.H. Golub, X. Wu, J.-Y. Yuan. SOR-like methods for augmented systems, BIT 41 (2001) 71-85.

[5] X.-Q. Jin, $M$-preconditioner for $M$-matrices, Appl. Math. Comput. 172 (2006) 701-707.

[6] S.-X. Miao, K. Wang, On generalized stationary iterative method for solving the saddle point problems, J. Appl. Math. Comput. 35 (2011) 459-468. 
[7] C.P. Pan, H.Y. Wang, On generalized preconditioned Hermitian and skew-Hermitian splitting methods for saddle point problems (in Chinese), J. Numer. Methods Comput. Appl. 32 (2011) 174-182.

[8] X. Shao, H. Shen, C. Li, T. Zhang, Generalized AOR method for augmented systems (in Chinese), J. Numer. Methods Comput. Appl. 27 (2006) 241-248. 